\title{
UNIFORM STABILITY FOR FRACTIONAL CAUCHY PROBLEMS AND APPLICATIONS
}

\author{
LUCIANO ABADIAS AND EDGARDO ALVAREZ
}

\begin{abstract}
In this paper we give uniform stable spatial bounds for the resolvent operator families of the abstract fractional Cauchy problem on $\mathbb{R}_{+}$. Such bounds allow to prove existence and uniqueness of $\mu$-pseudo almost automorphic $\epsilon$-mild regular solutions to the nonlinear fractional Cauchy problem in the whole real line. Finally, we apply our main results to the fractional heat equation with critical nonlinearities.
\end{abstract}

\section{INTRODUCTION}

In recent years, the study of fractional partial differential equations has growth considerably because those equations provide a useful framework to deal real-world problems in several disciplines as biology, chemistry, economics, engineering, medicine and physics. For example, fractional models describe the motion of a viscous fluid between moving surfaces ([27]), as well as the diffusion phenomena in special types of porous medium ([39]).

Let $A$ be a linear closed operator with dense domain defined on a Banach space $X$, and $0<\alpha<1$. We consider the nonlinear fractional Cauchy problem

$$
\left\{\begin{array}{l}
{ }_{C} D_{t}^{\alpha} u(t)=A u(t)+f(t, u(t)), \quad t \geq 0, \\
u(0)=u_{0} \in X
\end{array}\right.
$$

where $f: \mathbb{R}_{+} \times X \rightarrow X$ is a continuous function and ${ }_{C} D_{t}^{\alpha}$ denotes the fractional Caputo derivative. Under suitable conditions on the operator $A$ (sectoriality), it generates two operator families, $(S(t))_{t \geq 0}$ and $(R(t))_{t>0}$, called resolvent and integral resolvent. In such case, existence and uniqueness of mild solutions of the problem (1.1) depend on those operator families, see $[7,15,22]$ and references therein. These types of operator families are framed into the theory of solutions of abstract Volterra integral equations studied in the book of J. Prüss [43]. Also, algebraic, extension and subordination properties of them have been studied in $[2,3,6,7,13$, $28,29,30,31,35]$.

Historically, the importance of the asymptotic behaviour of the resolvent operator families is shown in many works in the literature. For the first order case, the uniform stability of a $C_{0}$-semigroup $\left(e^{t A}\right)_{t \geq 0}$ generated by $A$ is defined by the exponentially decay

$$
\left\|e^{t A}\right\| \leq M e^{-t a}, \quad t \geq 0
$$

2010 Mathematics Subject Classification. 34A08; 43A60; 47D06; 34G20.

Key words and phrases. Fractional Cauchy problem, uniform stability, $\mu$-pseudo almost automorphic functions, resolvent operator families, mild solutions.

L. Abadias is partially supported by Project MTM2016-77710-P, DGI-FEDER, of the MCYTS, Spain.

E. Alvarez is partially supported by Dirección de Gestión y Desarrollo Académico de la Universidad del Norte. 
where $M, a$ are positive constants. This theory has been deeply developed in a large series of monographs, see $[4,17,44]$ among others. Also, many authors have used the uniform stability of semigroups for specific problems. For example, Katznelson-Tzafriri type theorems for $C_{0^{-}}$ semigroups appear in the book of J. Van Neerven [44]. In [38], the stability of two-coupled systems of PDE's is shown. Recently, in order to face the third order Moore-Gibson-Thompson equation, the authors study such equation as a first order system whose associated operator matrix generates a exponentially stable semigroup on a Hilbert space, see [26].

In the book [40, Chapter 2, Section 6], A. Pazy shows uniform stable bounds for $C_{0}$-semigroups in the spaces constructed via the fractional powers of the generators. Particulary, when the spectrum of the operator $A$ satisfies that

$$
\sigma(-A) \subset\{\lambda \in \mathbb{C} \mid \operatorname{Re} \lambda>a\} \text { with } a>0,
$$

then

$$
\left\|(-A)^{\beta} e^{t A} x\right\| \leq M t^{-\beta} e^{-a t}\|x\|, \quad t>0, \beta>0, x \in X .
$$

It seems natural to ask about the uniform stability of the resolvent operator families for the fractional problems. In a general context, C. Lizama and V. Vergara give sufficient conditions to state exponential stability of resolvent families for the abstract Volterra integral equation, see [32]. Also, for the fractional case, it is known that the resolvent and the integral resolvent satisfy

$$
\|S(t)\| \leq M E_{\alpha, 1}\left(-a t^{\alpha}\right), \quad\|R(t)\| \leq M t^{\alpha-1} E_{\alpha, \alpha}\left(-a t^{\alpha}\right), \quad t>0,
$$

when the condition (1.2) holds for the spectrum of the generator, where $E_{\alpha, \beta}$ denotes the MittagLeffler functions. However, to the best of our knowledge, it seems newfangled to study the uniform stability of the resolvent operator families for the fractional Cauchy problem on the powers spaces.

Assuming conditions of sectoriality for an operator $-A$ such that $0 \in \rho(A)$ (resolvent set), we get that uniform stability for the resolvent families is given by the Prabhakar functions $E_{\alpha, \beta}^{\gamma}$, which are a generalization of Mittag-Leffler functions. In fact, we obtain that for all $x \in X$

$\|S(t) x\|_{X^{\beta}} \leq M t^{-\alpha \beta} E_{\alpha, 1-\alpha \beta}^{1-\beta}\left(-a t^{\alpha}\right)\|x\|, \quad\|R(t) x\|_{X^{\beta}} \leq M t^{\alpha(1-\beta)-1} E_{\alpha, \alpha(1-\beta)}^{1-\beta}\left(-a t^{\alpha}\right)\|x\|, \quad t>0$.

These functions were introduced by T.R. Prabhakar in 1971, see [41]. Nowadays, they are relevant in the study of fractional relaxation and diffusion phenomena, see $[18,24]$.

As an application, this spatial stability allows us to study the existence and uniqueness of mild solutions to the fractional problem in the whole real line

$$
D_{t}^{\alpha} u(t)=A u(t)+f(t, u(t)), \quad t \in \mathbb{R},
$$

where $f: \mathbb{R} \times X \rightarrow X$ is a continuous function with critical nonlinearities. More precisely, we will show that equation (1.3) has a unique $\mu$-pseudo almost automorphic $\epsilon$-mild regular solution.

The space of $\mu$-pseudo almost automorphic functions was introduced by Blot et al. in [10]. In that paper the authors gave a result on existence and uniqueness of mild solutions for the first order problem. On the other hand, De Andrade et al. [16] proved the existence and uniqueness of pseudo almost periodic $\epsilon$-mild regular solutions to the first order problem in the real line. We point out that the space of $\mu$-pseudo almost automorphic functions contains the space of pseudo almost periodic functions.

The paper is organized as follows. In Section 2, we present the Prabhakar functions and its relation with the Wright functions (Proposition 2.1). Also we prove that, for some specific 
parameters, the Prabhakar functions are integrable on $\mathbb{R}_{+}$(Proposition 2.3). In Section 3, we recall the notion of the operator families associated to the fractional Cauchy problem on $\mathbb{R}_{+}$, some properties, and the subordination generation formulas via $C_{0}$-semigroups. Mainly, we state the uniform stability for these operators families on the fractional power spaces defined by the generator when it has sectorial properties (Theorem 3.6). In Section 4, we prove existence and uniqueness of $\mu$-pseudo almost automorphic $\epsilon$-mild regular solutions to the fractional problem (1.3) in the real line. We illustrate it with an example. Finally, we include basic results about $\mu$-pseudo almost automorphic functions in Section 5 (Appendix), in order to get a self-contained paper.

\section{Mittag-Leffler, Prabhakar and scaled Wright functions}

In this section we present some connection between the Prabhakar and Wright functions which will be useful on the spatial bounds obtained in the next section.

Let $\gamma>0$, in the following we write $g_{\gamma}(t):=\frac{t^{\gamma-1}}{\Gamma(\gamma)}$ for $t>0$.

The Riemann-Liouville fractional derivative of order $0<\alpha<1$ of a function $u$ defined on $\mathbb{R}_{+}:=[0, \infty)$ is given by

$$
{ }_{R} D_{t}^{\alpha} u(t)=\frac{d}{d t}\left(g_{1-\alpha} * u\right)(t)=\frac{d}{d t} \int_{0}^{t} g_{1-\alpha}(t-s) u(s) d s, \quad t>0,
$$

and the Caputo fractional derivative of order $0<\alpha<1$ is defined by

$$
{ }_{C} D_{t}^{\alpha} u(t)=\left(g_{1-\alpha} * \frac{d}{d t} u\right)(t)=\int_{0}^{t} g_{1-\alpha}(t-s) u^{\prime}(s) d s, \quad t>0 .
$$

It is well known that ${ }_{C} D_{t}^{\alpha} u(t)={ }_{R} D_{t}^{\alpha} u(t)-\frac{t^{-\alpha}}{\Gamma(1-\alpha)} u(0)$ for $0<\alpha<1$.

Let $\alpha, \beta>0$. The Mittag-Leffler functions are given by

$$
E_{\alpha, \beta}(z)=\sum_{k=0}^{\infty} \frac{z^{k}}{\Gamma(\alpha k+\beta)}, \quad z \in \mathbb{C} .
$$

We write $E_{\alpha}(z):=E_{\alpha, 1}(z)$. They are the solutions of the classical fractional differential problems

$$
{ }_{C} D_{t}^{\alpha} E_{\alpha}\left(\omega t^{\alpha}\right)=\omega E_{\alpha}\left(\omega t^{\alpha}\right)
$$

and

$$
{ }_{R} D_{t}^{\alpha}\left(t^{\alpha-1} E_{\alpha, \alpha}\left(\omega t^{\alpha}\right)\right)=\omega t^{\alpha-1} E_{\alpha, \alpha}\left(\omega t^{\alpha}\right),
$$

for $0<\alpha<1$, under certain initial conditions. Fore more details see [7, 33, 36].

An extension of the Mittag-Leffler functions are the Prabhakar functions

$$
E_{\alpha, \beta}^{\gamma}(z)=\sum_{k=0}^{\infty} \frac{(\gamma)_{k}}{k ! \Gamma(\alpha k+\beta)} z^{k}, \quad z \in \mathbb{C}, \gamma>0
$$

where $(\gamma)_{k}=\gamma(\gamma+1) \cdots(\gamma+k-1)=\frac{\Gamma(k+\gamma)}{\Gamma(\gamma)}$ denotes de Pochhammer symbol. They are entire functions. Note that for $\gamma=1$ we recover the classical Mittag-Leffler functions. The Laplace transform of the above functions is

$$
\int_{0}^{\infty} e^{-\lambda t} t^{\beta-1} E_{\alpha, \beta}^{\gamma}\left(\omega t^{\alpha}\right) d t=\frac{\lambda^{\alpha \gamma-\beta}}{\left(\lambda^{\alpha}-\omega\right)^{\gamma}}, \quad \mathfrak{R e} \lambda>|\omega|^{1 / \alpha}
$$


For convenience, we denote

$$
e_{\alpha, \beta}^{\gamma}(t, \omega):=t^{\beta-1} E_{\alpha, \beta}^{\gamma}\left(-\omega t^{\alpha}\right), \quad \omega \in \mathbb{C} .
$$

It is a direct consequence by use of the Laplace transform that

$$
\left(g_{\delta} * e_{\alpha, \beta}^{\gamma}(\cdot, \omega)\right)(t)=e_{\alpha, \beta+\delta}^{\gamma}(t, \omega), \quad \delta>0 .
$$

These functions $e_{\alpha, \beta}^{\gamma}(\cdot, \omega)$ are locally integrable and completely monotonic under the conditions

$$
0<\alpha \leq 1, \quad 0<\alpha \gamma \leq \beta \leq 1, \quad \mathfrak{R e} \omega>0,
$$

see [12]. For more details about Prabhakar functions see [19, 27, 33].

The Wright function, denoted by $W_{\lambda, \mu}$, was introduced by E. Maitland Wright in a series of notes starting from 1933 in the framework of the theory of partitions, see [45]. This entire function is defined by

$$
W_{\lambda, \mu}(z):=\sum_{n=0}^{\infty} \frac{z^{n}}{n ! \Gamma(\lambda n+\mu)}, \quad \lambda>-1, \mu \in \mathbb{C} .
$$

Also, the Wright function can be represented through the following integral representation using the Hankel formula for the Gamma function,

$$
W_{\lambda, \mu}(z)=\frac{1}{2 \pi i} \int_{H a} \sigma^{-\mu} e^{\sigma+z \sigma^{-\lambda}} d \sigma, \quad \lambda>-1, \mu \geq 0, z \in \mathbb{C},
$$

where $H a$ denotes the Hankel path defined as a contour that begins at $t=-\infty-i a(a>0)$, encircles the branch cut that lies along the negative real axis, and ends up at $t=-\infty+i b$ $(b>0)$. For more details see [33, Appendix F].

Recently in [3], two-parameter Wright functions, called scaled Wright functions, are introduced in order to establish a general approach to face the subordination formulas for the solutions of the fractional abstract Cauchy problems. For $0<\alpha<1$ and $\beta \geq 0$, the scaled Wright functions $\psi_{\alpha, \beta}$ in two variables are defined by

$$
\psi_{\alpha, \beta}(t, s):=t^{\beta-1} W_{-\alpha, \beta}\left(-s t^{-\alpha}\right), \quad t>0, s \in \mathbb{C} .
$$

Note that using the change of variable $z=\frac{\sigma}{t}$, we get the integral representation

$$
\psi_{\alpha, \beta}(t, s)=\frac{1}{2 \pi i} \int_{H a} z^{-\beta} e^{t z-s z^{\alpha}} d z, \quad t, s>0 .
$$

The functions $\psi_{\alpha, \beta}$ are considered in the literature in some particular cases: for $\beta=1-\alpha$, it was introduced in [34, Formula (6.2)] and studied in [33, p. 257] and [7]; for $\beta=0$ it is the stable Lévy process of order $\alpha$, see [11] and [47, Chapter IX]. Several and interesting properties satisfied by these functions are studied in [3].

The following result generalizes [3, Theorem 3 (iii) and (vi)].

Proposition 2.1. Let $0<\alpha<1, \beta \geq 0$ and $\gamma>0$. Then

$$
\int_{0}^{\infty} e^{\omega s} g_{\gamma}(s) \psi_{\alpha, \beta}(t, s) d s=t^{\alpha \gamma+\beta-1} E_{\alpha, \alpha \gamma+\beta}^{\gamma}\left(\omega t^{\alpha}\right), \quad \omega \in \mathbb{C}, t>0 .
$$


Proof. First of all observe that by [3, Theorem 3 (iii) and (vi)] and the positivity of $\psi_{\alpha, \beta}$ ([3, Theorem 3 (i)]) the integral is convergent. So, it is enough to prove the result for $\omega \in \mathbb{R}$. Using Laplace transform for $\lambda>|\omega|^{1 / \alpha}$ and Fubini's Theorem one gets

$$
\int_{0}^{\infty} e^{-\lambda t} \int_{0}^{\infty} e^{\omega s} g_{\gamma}(s) \psi_{\alpha, \beta}(t, s) d s d t=\lambda^{-\beta} \int_{0}^{\infty} e^{\omega s} g_{\gamma}(s) e^{-\lambda^{\alpha} s} d s=\frac{\lambda^{-\beta}}{\left(\lambda^{\alpha}-\omega\right)^{\gamma}}
$$

By the uniqueness of the Laplace transform and (2.1) we conclude the result.

Remark 2.2. Note that by the above proposition, if $0<\alpha \leq 1,0<\alpha \gamma \leq \beta \leq 1$ and $a>0$, we have

$$
0 \leq e_{\alpha, \beta}^{\gamma}(t, a) \leq \int_{0}^{\infty} g_{\gamma}(s) \psi_{\alpha, \beta-\alpha \gamma}(t, s) d s=g_{\beta}(t) \rightarrow 0, \quad t \rightarrow \infty
$$

where we have applied $\left[3\right.$, Theorem 3 (vi)] and $\psi_{\alpha, \beta-\alpha \gamma}(t, s) \geq 0$ ([3, Theorem 3 (i)]).

The last result of this section shows that for $\gamma=\beta / \alpha$ and $a>0$, the Prabhakar functions $e_{\alpha, \beta}^{\beta / \alpha}(\cdot, a)$ are, not only decreasing, but also integrable on $\mathbb{R}_{+}$, and in such case the identity (2.1) is valid for $\mathfrak{R e} \lambda \geq 0$.

Proposition 2.3. Let $0<\alpha, \beta<1$ and $a>0$. Then

$$
\int_{0}^{\infty} e_{\alpha, \beta}^{\beta / \alpha}(t, a) d t=\frac{1}{a^{\beta / \alpha}} .
$$

Proof. The function $e_{\alpha, \beta}^{\beta / \alpha}(\cdot, a)$ is non-negative since the conditions of (2.2) are satisfied. Then we can apply Fubini's Theorem, and by Proposition 2.1 we have

$$
\begin{aligned}
\int_{0}^{\infty} e_{\alpha, \beta}^{\beta / \alpha}(t, a) d t & =\int_{0}^{\infty} \int_{0}^{\infty} e^{-a s} g_{\beta / \alpha}(s) \psi_{\alpha, 0}(t, s) d s d t \\
& =\int_{0}^{\infty} \int_{0}^{\infty} e^{-a s} g_{\beta / \alpha}(s) t^{-1} W_{-\alpha, 0}\left(-s t^{-\alpha}\right) d s d t \\
& =\int_{0}^{\infty} g_{\beta / \alpha}(u) W_{-\alpha, 0}(-u) \int_{0}^{\infty} e^{-a u t^{\alpha}} t^{\beta-1} d t d u \\
& =\frac{1}{\alpha a^{\beta / \alpha}} \int_{0}^{\infty} u^{-1} W_{-\alpha, 0}(-u) d u=\frac{1}{a^{\beta / \alpha}},
\end{aligned}
$$

where we have used the Laplace transform of $t^{\beta-1}$ and that $\int_{0}^{\infty} u^{-1} W_{-\alpha, 0}(-u) d u=\alpha([33$, (F.33)]).

\section{Resolvent FAmilies}

From now on we denote by $\mathcal{B}(X)$ the space of all linear and bounded operators on the Banach space $X$. Let $A$ be a linear closed operator with dense domain defined on $X$. If the operator $A$ generates a resolvent and an integral resolvent operators families, $(S(t))_{t \geq 0}$ and $(R(t))_{t>0}$ respectively, it is well-known that the solutions of the fractional abstract Cauchy problem for $0<\alpha<1$,

$$
\left\{\begin{array}{l}
{ }_{C} D_{t}^{\alpha} u(t)=A u(t)+f(t, u(t)), \quad t \geq 0 \\
u(0)=u_{0} \in X
\end{array}\right.
$$


where $f: \mathbb{R}_{+} \times X \rightarrow X$ is a continuous function, satisfy

$$
u(t)=S(t) u_{0}+\int_{0}^{t} R(t-s) f(s, u(s)) d s, \quad t \geq 0 .
$$

It is a simple check by use of the Laplace transform. Usually, it is not possible to ensure the existence of strong solution for the fractional differential problem (3.1), and therefore we look for mild solutions for the associated Volterra integral equation (3.2). In many cases to do this (it depends on the techniques), it is useful to know the spatial bounds for the operator families that appear in the integral equation. In this section we will focus on studying such bounds with stable decay.

Definition 3.1. [43, Definition 1.3] A family $(S(t))_{t \geq 0} \subset \mathcal{B}(X)$ of bounded linear operators on $X$ is called a resolvent if the following conditions are satisfied.

(i) $S(t)$ is strongly continuous on $\mathbb{R}_{+}$and $S(0)=I$.

(ii) $S(t) D(A) \subset D(A)$ and $A S(t) x=S(t) A x$ for all $x \in D(A)$ and $t \geq 0$.

(iii) The equation

$$
S(t) x=x+\int_{0}^{t} g_{\alpha}(t-s) A S(s) x d s \quad \text { for all } x \in D(A), \quad t \geq 0,
$$

holds.

A resolvent is unique, if it exists. In such case, we call the operator $A$ the generator of the resolvent $(S(t))_{t \geq 0}$. In addition, $\left(g_{\alpha} * S\right)(t) x \in D(A)$ for all $x \in X$ and $t \geq 0$ ([43, Proposition $1.1])$.

Definition 3.2. A family $(R(t))_{t>0} \subset \mathcal{B}(X)$ of bounded linear operators on $X$ is called an integral resolvent if the following conditions are satisfied.

(i) $R(t)$ is strongly continuous on $(0, \infty)$ and $\lim _{t \rightarrow 0} \frac{R(t)}{g_{\alpha}(t)} x=x$ for all $x \in X$.

(ii) $R(t) D(A) \subset D(A)$ and $A R(t) x=R(t) A x$ for all $x \in D(A)$ and $t>0$.

(iii) The equation

$$
R(t) x=g_{\alpha}(t) x+\int_{0}^{t} g_{\alpha}(t-s) A R(s) x d s \quad \text { for all } x \in D(A), \quad t>0,
$$

holds.

Remark 3.3. Definition 3.2 corresponds to a slight modification to those given by Prüss [43, Definition 1.6] where ( $i$ ) is replaced by local integrability instead of strong continuity and (iii) is slightly different. We observe that the given condition near zero instead of the most common $R(0)=c I$, with $c$ the value of a corresponding kernel in zero, allows the treatment of singular kernels $g_{\alpha}$. The integral resolvent is unique, if it exists, and $A$ is the generator of the integral resolvent $(R(t))_{t>0}$. Also, $\left(g_{\alpha} * R\right)(t) x \in D(A)$ for all $x \in X$ and $t>0$. See also Li and Peng [30]. In a general context, both the resolvent and integral resolvent have been studied in different papers $([1,2,30,31,35,43])$. 
Remark 3.4. It is an easy computation that $1=g_{\alpha} * g_{1-\alpha}$. So if there exists an integral resolvent $(R(t))_{t>0}$ generated by $A$, then

$$
S(t) x:=\left(g_{1-\alpha} * R\right)(t) x=\int_{0}^{t} g_{1-\alpha}(t-s) R(s) x d s, \quad x \in X, \quad t>0,
$$

is a resolvent generated by $A$. Indeed, it is clear that the conditions (ii) and (iii) of Definition 3.1 are satisfied. Also, let $x \in X$ and $\varepsilon>0$, by Definition 3.2 (i), there exists $\delta>0$ such that

$$
\left\|\frac{R(s) x-g_{\alpha}(s) x}{g_{\alpha}(s)}\right\|<\varepsilon, \quad s \in(0, \delta) .
$$

So,

$$
\begin{aligned}
\|S(t) x-x\| & =\left\|\int_{0}^{t} g_{1-\alpha}(t-s)\left(R(s) x-g_{\alpha}(s) x\right) d s\right\| \\
& <\varepsilon \int_{0}^{t} g_{1-\alpha}(t-s) g_{\alpha}(s) d s=\varepsilon, \quad t \in(0, \delta) .
\end{aligned}
$$

By continuity, we can define $S(0)=I$. In addition,

$$
\left(g_{1-\alpha} * R\right)(t) x=x+A\left(g_{1-\alpha} * g_{\alpha} * R\right)(t) x=x+A \int_{0}^{t} R(s) x, \quad x \in X,
$$

therefore $S(\cdot) x=\left(g_{1-\alpha} * R\right)(\cdot) x \in C^{1}\left(\mathbb{R}_{+}, X\right)$ for $x \in D(A)$. Using (3.3), (3.4) and (3.5) we get

and

$$
\begin{gathered}
{ }_{R} D_{t}^{\alpha} R(t) x=A R(t) x, \quad x \in D(A), \\
\frac{d}{d t} S(t) x=A R(t) x, \quad x \in D(A),
\end{gathered}
$$

$$
{ }_{C} D_{t}^{\alpha} S(t) x=A S(t) x, \quad x \in D(A) .
$$

We recall the following notion of operator theory.

Definition 3.5. (Sectorial operator) Let $B: D(B) \subset X \rightarrow X$ be a linear operator. We say that $B$ is sectorial of angle $\vartheta \in[0, \pi)$ if

$$
\sigma(B) \subset \overline{\Sigma_{\vartheta}}
$$

and

$$
\left\|\lambda(\lambda-B)^{-1}\right\| \leq M_{\varphi}, \quad \lambda \in \mathbb{C} \backslash \overline{\Sigma_{\varphi}}, \text { for all } \varphi \in(\vartheta, \pi)
$$

where $\sigma(B)$ denotes the spectrum of $B$, and $\Sigma_{\vartheta}:=\{\lambda \in \mathbb{C}: \lambda \neq 0,|\arg (\lambda)|<\vartheta\}$ for $\vartheta \in(0, \pi]$ and $\Sigma_{0}:=(0, \infty)$ if $\vartheta=0$.

If $b+B$ is sectorial of angle $\vartheta$ with $b \in \mathbb{R}$, we say that $B$ is b-sectorial of angle $\vartheta$.

For more information about the theory of sectorial operators we refer the reader to the monograph [23].

It is well known that if an operator $-A$ is closed, densely defined and sectorial of angle $\vartheta \in$ $[0, \pi / 2)$, then $A$ generates a bounded holomorphic $C_{0}$-semigroup $(T(t))_{t \geq 0}$ on $X$. The converse is also true. Moreover, if $-A$ is $-a$-sectorial of angle $\vartheta \in[0, \pi / 2)$ with $a>0$, then $A$ generates a uniform stable bounded holomorphic $C_{0}$-semigroup, that is,

$$
\|T(t) x\| \leq M e^{-a t}\|x\| .
$$


In such case, the semigroup has the following integral representation formula in terms of the resolvent operator,

$$
T(t) x=\int_{-a+\gamma} e^{\lambda t}(\lambda-A)^{-1} x d \lambda, \quad t>0, x \in X,
$$

where $\gamma$ is the complex Hankel's path given by

$$
\gamma=\gamma_{r, \omega}:=\left\{\lambda e^{i \omega}: \lambda \geq r\right\} \cup\left\{r e^{i \varphi}: \varphi \in(-\omega, \omega)\right\} \cup\left\{\lambda e^{-i \omega}: \lambda \geq r\right\},
$$

oriented counterclockwise, with $r>0$ and $\omega \in(\pi / 2, \pi-\vartheta)$. It follows that

$$
\|A T(t) x\| \leq M \frac{e^{-a t}}{t}\|x\|, \quad t>0, x \in X .
$$

Observe that if $-A$ is closed, densely defined and sectorial of angle $\vartheta \in[0, \pi / 2)$ with $0 \in \rho(A)$, then, since the spectrum is closed, we have that the generated $C_{0}$-semigroup is uniform stable. For example, the Laplace operator $-\Delta$ on $L^{2}(0, T)$, with $T>0$, is sectorial of angle 0 , and the eigenvalues are strictly positive. In this case, $-\Delta$ is $-a$-sectorial for all $0<a<\lambda_{1}$, where $\lambda_{1}$ is the first eigenvalue (see [25, Exercise p. 23]). For more details see [25, Chapter 1].

From now on, we suppose that $-A$ is a $-a$-sectorial of angle $\vartheta \in[0, \pi / 2)$ with $a>0$. Then one can define the negative fractional powers of the operator

$$
(-A)^{-\beta} x=\frac{1}{\Gamma(\beta)} \int_{0}^{\infty} s^{\beta-1} T(s) x d s, \quad \beta>0, \quad x \in X .
$$

This operator is injective, so we define $(-A)^{\beta}$ as the inverse of $(-A)^{-\beta}$ with domain $D\left((-A)^{\beta}\right)=$ $r g\left(-(A)^{-\beta}\right)$. For each $\beta \geq 0$, we will denote the fractional spaces

$$
X^{\beta}=D\left((-A)^{\beta}\right)
$$

with the graph norm $\|x\|_{X^{\beta}}=\left\|(-A)^{\beta} x\right\|$. In such case, the exponentially stable bounded holomorphic $C_{0}$-semigroup satisfies

$$
\|T(t) x\|_{X^{\beta}} \leq M g_{1-\beta}(t) e^{-a t}\|x\|, \quad 0 \leq \beta<1,
$$

and the resolvent operator

$$
\left\|(-A)^{\beta}(\lambda-A)^{-1}\right\| \leq M|\lambda+a|^{\beta-1}, \quad \lambda \in-a+\Sigma_{\pi-\vartheta} .
$$

For more details see [25, Chapter 1$]$.

In [3], the authors studied subordination formulas to get resolvent and integral resolvent families via $C_{0}$-semigroups, as particular cases of a general framework of subordination theory. For that purpose, the scaled Wright functions are needed. If $(T(t))_{t \geq 0}$ is the semigroup generated by $A$, then

$$
S(t) x=\int_{0}^{\infty} \psi_{\alpha, 1-\alpha}(t, s) T(s) x d s, \quad R(t) x=\int_{0}^{\infty} \psi_{\alpha, 0}(t, s) T(s) x d s, \quad t>0, x \in X,
$$

are the resolvent and the integral resolvent generated by $A$. The following result shows that, in fact, they are uniformly stable in the fractional spatial spaces $X^{\beta}$ for $0 \leq \beta<1$, that is, $\lim _{t \rightarrow \infty}\|S(t) x\|_{X^{\beta}}=0$ and $\lim _{t \rightarrow \infty}\|R(t) x\|_{X^{\beta}}=0$ for $x \in X$. Moreover, the integral resolvent $(R(t))_{t>0}$ is integrable on $\mathbb{R}_{+}$. The proof is a straightforward consequence of (3.8), (3.7) and Proposition 2.1.

Theorem 3.6. Let $-A$ be $a-a$-sectorial of angle $\vartheta \in[0, \pi / 2)$ with $a>0$, and $0 \leq \beta<1$. For $x \in X$ it follows

(i) $\|S(t) x\|_{X^{\beta}} \leq M e_{\alpha, 1-\alpha \beta}^{1-\beta}(t, a)\|x\|, \quad t>0$. 
(ii) $\|R(t) x\|_{X^{\beta}} \leq M e_{\alpha, \alpha(1-\beta)}^{1-\beta}(t, a)\|x\|, \quad t>0$.

Remark 3.7. If we take $\beta=0$ in the above result we have

(i) $\|S(t) x\| \leq M E_{\alpha, 1}\left(-a t^{\alpha}\right)\|x\|, \quad t>0$.

(ii) $\|R(t) x\| \leq M t^{\alpha-1} E_{\alpha, \alpha}\left(-a t^{\alpha}\right)\|x\|, \quad t>0$.

The estimate given in part (ii) of this Remark appears in [37].

Remark 3.8. Observe that Theorem 3.6 is not valid for $\beta=1$. To get the spatial bounds in this special case, we use (3.6). Indeed, let $x \in X$ and $t>0$, one gets

$$
\begin{aligned}
\|R(t) x\|_{X^{1}} & \leq M \int_{0}^{\infty} \psi_{\alpha, 0}(t, s) \frac{e^{-a s}}{s} d s \\
& =M t^{-1} \int_{0}^{\infty} W_{-\alpha, 0}\left(-s t^{-\alpha}\right) \frac{e^{-a s}}{s} d s \\
& =M \alpha t^{-1} \int_{0}^{\infty} t^{-\alpha} W_{-\alpha, 1-\alpha}\left(-s t^{-\alpha}\right) e^{-a s} d s \\
& =M \alpha t^{-1} \int_{0}^{\infty} \psi_{\alpha, 1-\alpha}(t, s) e^{-a s} d s \\
& =M \alpha t^{-1} E_{\alpha, 1}\left(-a t^{\alpha}\right),
\end{aligned}
$$

where $[33,(\mathrm{~F} .11)]$ and $[3$, Theorem 3 (iii)] have been used. Note that for the case $0 \leq \beta<1$, the spatial bound obtained in Theorem 3.6 implies the integrability of the family $(R(t))_{t>0}$, however this does not happen for case $\beta=1$ because $t^{-1} E_{\alpha, 1}\left(-a t^{\alpha}\right)$ is not integrable at zero. This can be compared to the semigroup case, see inequalities (3.6) and (3.7).

For the resolvent family $(S(t))_{t \geq 0}$, the subordination formula (3.8) does not give information to get the spatial bound in case $\beta=1$. Even doing use of the bound of $(R(t))_{t>0}$, we can not obtain the corresponding one for $(S(t))_{t \geq 0}$ with the relation $S=g_{1-\alpha} * R$ since $t^{-1} E_{\alpha, 1}\left(-a t^{\alpha}\right)$ is not integrable at zero.

From Theorem 3.6 one gets the following generalized spatial bounds.

Corollary 3.9. Let $-A$ be $a$-a-sectorial of angle $\vartheta \in[0, \pi / 2)$ with $a>0$, and $0 \leq \theta<\beta \leq 1$. For $x \in X^{\beta}$ we have

(i) $\|S(t) x\|_{X^{1+\theta}} \leq M e_{\alpha, 1-\alpha(1+\theta-\beta)}^{\beta-\theta}(t, a)\|x\|_{X^{\beta}}, \quad t>0$.

(ii) $\|R(t) x\|_{X^{1+\theta}} \leq M e_{\alpha, \alpha(\beta-\theta)}^{\beta-\theta}(t, a)\|x\|_{X^{\beta}}, \quad t>0$.

Remark 3.10. If $-A$ is -a-sectorial of angle $\vartheta \in[0, \pi / 2)$ with $a>0$, then the associated exponentially stable bounded holomorphic $C_{0}$-semigroup $(T(t))_{t \geq 0}$ is characterized by the strong continuity and its Laplace transform, that is,

$$
(\lambda-A)^{-1} x=\int_{0}^{\infty} e^{-\lambda t} T(t) x d t, \quad x \in X, \mathfrak{R e} \lambda>-a .
$$

For more details see [4]. The same happens for the resolvent and the integral resolvent, that is, $(S(t))_{t>0}$ and $(R(t))_{t>0}$ are characterized by the strong continuity and their Laplace transforms,

$$
\lambda^{\alpha-1}\left(\lambda^{\alpha}-A\right)^{-1} x=\int_{0}^{\infty} e^{-\lambda t} S(t) x d t, \quad x \in X, \mathfrak{R e} \lambda>0,
$$




$$
\left(\lambda^{\alpha}-A\right)^{-1} x=\int_{0}^{\infty} e^{-\lambda t} R(t) x d t, \quad x \in X, \mathfrak{R e} \lambda \geq 0 .
$$

This can be proved by subordination, or as a slightly variation of [31, Proposition 3.1].

Remark 3.11. In [15] and [22] the authors use the spatial bounds for the resolvent and integral resolvent families generated by an operator $-A$ which is sectorial of angle $\vartheta \in[0, \pi / 2)$, in order to get solutions for (3.1) where $f$ has critical nonlinearities. It would be interesting to study what happens if we consider $a-a$-sectorial operator, with $a>0$, of angle $\vartheta \in[0, \pi / 2)$, and how this affects to the solutions in the critical case. It seems that the solutions would behave locally near to the origin as the Prabhakar functions do it.

In this paper our aim is other. We will use the integrability of the integral resolvent associated to -a-sectorial operators, where $a>0$, in order to get solutions in the spaces of $\mu$-pseudo almost automorphic functions to the fractional Cauchy problem on the real line with critical nonlinearities.

\section{4. $\mu$-PSEUdo ALMOST AUTOMORPHIC $\epsilon$-MILD REGUlAR SOLUTIONS}

In what follows we will use the notation and some basic results given in the Appendix. We encourage to the reader to see that section.

Let $0<\alpha<1$ and $A$ be a linear closed operator with dense domain defined on a Banach space $X$. In order to show the relevance of the spatial bounds obtained in the previous section, we are going to study $\mu$-pseudo almost automorphic solutions for the fractional problem on the real line

$$
D_{t}^{\alpha} u(t)=A u(t)+f(t, u(t)), \quad t \in \mathbb{R}
$$

where $-A$ is a $-a$-sectorial operator, with $a>0$, of angle $\vartheta \in[0, \pi / 2)$ on the Banach space $X$, and $f: \mathbb{R} \times X \rightarrow X$ is a continuous function with critical nonlinearities. Now, the fractional derivative of order $0<\alpha<1$ of a function $u: \mathbb{R} \rightarrow X$ is given by

$$
D_{t}^{\alpha} u(t)=\frac{d}{d t}\left(g_{1-\alpha} * u\right)(t)=\frac{d}{d t} \int_{-\infty}^{t} g_{1-\alpha}(t-s) u(s) d s
$$

and it represents the Weyl, Caputo, Riemann-Liouville and Grunwald-Letnikov, which coincide under some regularity assumptions, see [42].

Let $u: \mathbb{R} \rightarrow X$ be a solution of (4.1) and we denote by $\mathcal{F} u$ the Fourier transform of $u$, that is,

$$
(\mathcal{F} u)(\eta)=\int_{-\infty}^{\infty} e^{-i \eta t} u(t) d t, \quad \eta \in \mathbb{R}
$$

whenever the integral converges. In a formal way, if we assume regularity properties to apply Fourier transform to (4.1), we have that

$$
(\mathcal{F} u)(\eta)=\left((i \eta)^{\alpha}-A\right)^{-1}(\mathcal{F} f(\cdot, u(\cdot)))(\eta), \quad \eta \in \mathbb{R},
$$

since $(i \eta)^{\alpha} \in \rho(A)$ by the $-a$-sectoriality of $-A$, with $a>0$. Therefore, by the above comment and (3.9) we state the following definition.

Definition 4.1. Let $\epsilon>0$. We say that a function $u: \mathbb{R} \rightarrow X^{1}$ such that $u \in C\left(\mathbb{R}, X^{1+\varepsilon}\right)$ is an $\epsilon$-mild regular solution of (4.1) if it satisfies

$$
u(t)=\int_{-\infty}^{t} R(t-s) f(s, u(s)) d s, \quad t \in \mathbb{R} .
$$


Now, we recall the notion of $\epsilon$-regular map due to Arrieta and Carvalho [5, Definition 2] to establish the nonlinearities in the equation (4.1).

Definition 4.2. For $\epsilon>0$ we say that a map $g$ is an $\epsilon$-regular map relative to the pair $\left(X^{1}, X^{0}\right)$ if there exist $\rho>1, \gamma(\epsilon)$ with $\rho \epsilon \leq \gamma(\epsilon)<1$, and a positive constant $c$, such that $g: X^{1+\epsilon} \rightarrow X^{\gamma(\epsilon)}$ and

$$
\|g(x)-g(y)\|_{X^{\gamma(\epsilon)}} \leq c\left(1+\|x\|_{X^{1+\epsilon}}^{\rho-1}+\|y\|_{X^{1+\epsilon}}^{\rho-1}\right)\|x-y\|_{X^{1+\epsilon}}
$$

for all $x, y \in X^{1+\epsilon}$.

In this work we consider the following class of nonlinearities: with $\epsilon, \gamma(\varepsilon), \rho$, and $c$ positive constants, the class $\mathcal{F}(\epsilon, \gamma(\varepsilon), \rho, c)$ is defined as the family of functions $f$ such that, for $t \in \mathbb{R}$, $f(t, \cdot)$ is an $\epsilon$-regular map relative to the pair $\left(X^{1}, X^{0}\right)$, satisfying

$$
\|f(t, x)-f(t, y)\|_{X^{\gamma(\epsilon)}} \leq c\left(1+\|x\|_{X^{1+\epsilon}}^{\rho-1}+\|y\|_{X^{1+\epsilon}}^{\rho-1}\right)\|x-y\|_{X^{1+\epsilon}},
$$

$$
\|f(t, x)\|_{X^{\gamma(\epsilon)}} \leq c\left(1+\|x\|_{X^{1+\epsilon}}^{\rho}\right)
$$

for all $x, y \in X^{1+\epsilon}$.

Remark 4.3. Suppose that $f: \mathbb{R} \times X^{1+\epsilon} \rightarrow X^{\gamma(\epsilon)}$ belongs to the class $\mathcal{F}(\epsilon, \gamma(\varepsilon), \rho, c)$. We claim that $f$ satisfies Condition $(C)$ in Theorem 5.13 (see Appendix). Indeed, let $B$ be a bounded set of $X^{1+\epsilon}$. Then there exists $M_{0}>0$ such that $\|y\|_{X^{1+\epsilon}} \leq M_{0}$ for all $y \in B$. Therefore

$$
\|f(t, y)\|_{X^{\gamma(\epsilon)}} \leq c\left(1+\|y\|_{X^{1+\epsilon}}^{\rho}\right) \leq c\left(1+M_{0}^{\rho}\right), \quad(t, y) \in \mathbb{R} \times B .
$$

It follows that $f$ is bounded on $\mathbb{R} \times B$.

In the remaining of this section we assume that $\mu \in \mathcal{M}$ satisfying Condition $(H)$ (see Appendix).

Now, we state a technical lemma of convolution type, which will be used in the main result of the section.

Lemma 4.4. Let $h \in P A A\left(X^{\gamma(\epsilon)}, \mu\right)$. Then for every $0 \leq \theta<\gamma(\epsilon)$ we have that

$$
H(t):=\int_{-\infty}^{t} R(t-s) h(s) d s \in P A A\left(X^{1+\theta}, \mu\right) .
$$

Proof. Let $h=f+\phi$ with $f \in A A\left(X^{\gamma(\epsilon)}\right)$ and $\phi \in \mathcal{E}\left(X^{\gamma(\epsilon)}, \mu\right)$. Then we can consider

$$
H(t):=F(t)+\Phi(t)
$$

where

$$
F(t):=\int_{-\infty}^{t} R(t-s) f(s) d s, \quad \Phi(t):=\int_{-\infty}^{t} R(t-s) \phi(s) d s .
$$

First, we prove that $F \in A A\left(X^{1+\theta}\right)$. Indeed, since $f \in A A\left(X^{\gamma(\epsilon)}\right)$, then given a sequence $\left(s_{n}^{\prime}\right)_{n \in \mathbb{N}}$ of real numbers, there exists a subsequence $\left(s_{n}\right)_{n \in \mathbb{N}}$ and a function $g$ such that

$$
\left\|f\left(t+s_{n}\right)-g(t)\right\|_{X^{\gamma(\epsilon)}} \rightarrow 0 \quad(n \rightarrow \infty),
$$

and

$$
\left\|g\left(t-s_{n}\right)-f(t)\right\|_{X^{\gamma(\epsilon)}} \rightarrow 0 \quad(n \rightarrow \infty) .
$$


This means that for $\delta>0$ given, there exists $N \in \mathbb{N}$ such that $\left\|f\left(t+s_{n}\right)-g(t)\right\|_{X^{\gamma(\epsilon)}}<\delta$ provided $n>N$. Let

$$
G(t):=\int_{-\infty}^{t} R(t-s) g(s) d s
$$

Note that

$$
F\left(t+s_{n}\right)=\int_{-\infty}^{t} R(t-s) f\left(s+s_{n}\right) d s .
$$

Then by Corollary 3.9 we have that for $n>N$

$$
\begin{aligned}
\left\|F\left(t+s_{n}\right)-G(t)\right\|_{X^{1+\theta}} & \leq \int_{-\infty}^{t}\left\|R(t-s)\left[f\left(s+s_{n}\right)-g(s)\right]\right\|_{X^{1+\theta}} d s \\
& \leq M \int_{-\infty}^{t} e_{\alpha, \alpha(\gamma(\epsilon)-\theta)}^{\gamma(\epsilon)-\theta}(t-s, a)\left\|f\left(s+s_{n}\right)-g(s)\right\|_{X^{\gamma(\epsilon)}} d s \\
& <\frac{M \delta}{a^{\gamma(\epsilon)-\theta}},
\end{aligned}
$$

where we have used Proposition 2.3. Hence $\left\|F\left(t+s_{n}\right)-G(t)\right\|_{X^{1+\theta}} \rightarrow 0$ as $n \rightarrow \infty$. Analogously we prove that $\left\|G\left(t-s_{n}\right)-F(t)\right\|_{X^{1+\theta}} \rightarrow 0$ as $n \rightarrow \infty$. It follows that $F \in A A\left(X^{1+\theta}\right)$.

The next step consists in to prove that $\Phi \in \mathcal{E}\left(X^{1+\theta}, \mu\right)$, that is,

$$
\lim _{T \rightarrow \infty} \frac{1}{\mu([-T, T])} \int_{-T}^{T}\|\Phi(t)\|_{X^{1+\theta}} d \mu(t)=0 .
$$

Indeed, by Corollary 3.9 we deduce that

$$
\|\Phi(t)\|_{X^{1+\theta}} \leq M \int_{0}^{\infty} e_{\alpha, \alpha(\gamma(\epsilon)-\theta)}^{\gamma(\epsilon)-\theta}(s, a)\|\phi(t-s)\|_{X^{\gamma(\epsilon)}} d s .
$$

Then by Fubini's Theorem

$\frac{1}{\mu([-T, T])} \int_{-T}^{T}\|\Phi(t)\|_{X^{1+\theta}} d \mu(t) \leq \int_{0}^{\infty} e_{\alpha, \alpha(\gamma(\epsilon)-\theta)}^{\gamma(\epsilon)-\theta}(s, a)\left(\frac{1}{\mu([-T, T])} \int_{-T}^{T}\|\phi(t-s)\|_{X^{\gamma(\epsilon)}} d \mu(t)\right) d s$.

Note that by Corollary 2.3 we get

$e_{\alpha, \alpha(\gamma(\epsilon)-\theta)}^{\gamma(\epsilon)-\theta}(s, a) \frac{1}{\mu([-T, T])} \int_{-T}^{T}\|\phi(t-s)\|_{X^{\gamma(\epsilon)}} d \mu(t) \leq e_{\alpha, \alpha(\gamma(\epsilon)-\theta)}^{\gamma(\epsilon)-\theta}(s, a) \sup _{\sigma \in \mathbb{R}}\|\phi(\sigma)\|_{X^{\gamma(\epsilon)}} \in L^{1}\left(\mathbb{R}_{+}\right)$.

On the other hand, by the translation invariance of $\mathcal{E}\left(X^{\gamma(\epsilon)}, \mu\right)$ (see Theorem 5.6) one gets

$$
\lim _{T \rightarrow \infty} \frac{1}{\mu([-T, T])} \int_{-T}^{T}\|\phi(t-s)\|_{X^{\gamma(\epsilon)}} d \mu(t)=0, \quad s \in \mathbb{R}_{+} .
$$

Hence, it follows from Dominated Convergence Theorem that $\Phi \in \mathcal{E}\left(X^{1+\theta}, \mu\right)$, and we conclude that $H \in P A A\left(X^{1+\theta}, \mu\right)$.

Finally, we present the main result of this section.

Theorem 4.5. Let $f \in \mathcal{F}(\epsilon, \gamma(\varepsilon), \rho, c) \cap P A A_{U}\left(X^{1+\epsilon}, X^{\gamma(\epsilon)}, \mu\right)$. If the constant c satisfies

$$
M c L(r)<a^{\gamma(\epsilon)-\epsilon}, \text { where } L(r)=\max \left\{r^{-1}+r^{\rho-1}, 1+2 r^{\rho-1}\right\}
$$

then problem (4.1) has a unique $\mu$-pseudo almost automorphic $\epsilon$-regular mild solution. Moreover, this solution satisfies $u \in P A A\left(X^{1+\theta}, \mu\right)$, for every $0 \leq \theta<\gamma(\epsilon)$. 
Proof. Let $T: P A A\left(X^{1+\epsilon}, \mu\right) \rightarrow P A A\left(X^{1+\theta}, \mu\right)$ given by

$$
(T u)(t)=\int_{-\infty}^{t} R(t-s) f(s, u(s)) d s,
$$

for each $0 \leq \theta<\gamma(\epsilon)$

We divide the proof into 3 steps.

Step 1. First we see that $T$ is well-defined. Let $u \in P A A\left(X^{1+\epsilon}, \mu\right)$. Since $f \in P A A_{U}\left(X^{1+\epsilon}, X^{\gamma(\epsilon)}, \mu\right)$ and $u \in P A A\left(X^{1+\epsilon}, \mu\right)$ then by Theorem 5.13 we get $f(\cdot, u(\cdot)) \in P A A\left(X^{\gamma(\epsilon)}, \mu\right)$, where we have used Remark 4.3. On the other hand, by Lemma 4.4 we have $T u \in P A A\left(X^{1+\theta}, \mu\right)$.

Step 2. Let $u \in P A A\left(X^{1+\epsilon}, \mu\right)$, we see that $T u: \mathbb{R} \rightarrow X^{1+\theta}$ is bounded and continuous. Let $t \in \mathbb{R}$ and $h>0$. Then

$$
\begin{aligned}
\|(T u)(t+h)-(T u)(t)\|_{X^{1+\theta}} & =\left\|\int_{-\infty}^{t+h} R(t+h-s) f(s, u(s)) d s-\int_{-\infty}^{t} R(t-s) f(s, u(s)) d s\right\|_{X^{1+\theta}} \\
& =\| \int_{-\infty}^{t}[R(t+h-s)-R(t-s)] f(s, u(s)) d s \\
& +\int_{t}^{t+h} R(t+h-s) f(s, u(s)) d s \|_{X^{1+\theta}} \\
& \leq \int_{0}^{\infty}\|[R(s+h)-R(s)] f(t-s, u(t-s))\|_{X^{1+\theta}} d s \\
& +\int_{t}^{t+h}\|R(t+h-s) f(s, u(s))\|_{X^{1+\theta}} d s=: I_{1}+I_{2} .
\end{aligned}
$$

We claim that $I_{1} \rightarrow 0$ as $h \rightarrow 0^{+}$. In fact, since $(R(t))_{t>0}$ is strongly continuous on $X$ we have that $\|[R(s+h)-R(s)] f(t-s, u(t-s))\|_{X^{1+\theta}} \rightarrow 0$ as $h \rightarrow 0^{+}$. Also, by Corollary 3.9 and Proposition 2.3 we have

$$
\begin{aligned}
\|[R(s+h)-R(s)] f(t-s, u(t-s))\|_{X^{1+\theta}} & \leq M c\left[e_{\alpha, \alpha(\gamma(\epsilon)-\theta)}^{\gamma(\epsilon)-\theta}(s+h, a)+e_{\alpha, \alpha(\gamma(\epsilon)-\theta)}^{\gamma(\epsilon)-\theta}(s, a)\right] \\
& \times\left(1+\sup _{\sigma \in \mathbb{R}}\|u(\sigma)\|_{X^{1+\epsilon}}^{\rho}\right) \\
& \leq 2 M c e_{\alpha, \alpha(\gamma(\epsilon)-\theta)}^{\gamma(\epsilon)-\theta}(s, a)\left(1+\sup _{\sigma \in \mathbb{R}}\|u(\sigma)\|_{X^{1+\epsilon}}^{\rho}\right) \in L^{1}\left(\mathbb{R}_{+}\right),
\end{aligned}
$$

where we have used that $e_{\alpha, \alpha(\gamma(\epsilon)-\theta)}^{\gamma(\epsilon)-\theta}(\cdot, a)$ is decreasing because it is completely monotonic, see (2.2). It follows from the Dominated Convergence Theorem that $I_{1} \rightarrow 0$ as $h \rightarrow 0^{+}$. Now,

$$
\begin{aligned}
I_{2} & \leq M c\left(1+\sup _{\sigma \in \mathbb{R}}\|u(\sigma)\|_{X^{1+\epsilon}}^{\rho}\right) \int_{t}^{t+h} e_{\alpha, \alpha(\gamma(\epsilon)-\theta)}^{\gamma(\epsilon)-\theta}(t+h-s, a) d s \\
& =M c\left(1+\sup _{\sigma \in \mathbb{R}}\|u(\sigma)\|_{X^{1+\epsilon}}^{\rho}\right) \int_{0}^{h} e_{\alpha, \alpha(\gamma(\epsilon)-\theta)}^{\gamma(\epsilon)-\theta}(\tau, a) d \tau \rightarrow 0
\end{aligned}
$$

as $h \rightarrow 0^{+}$. Furthermore,

$$
\|(T u)(t)\|_{X^{1+\theta}} \leq M c\left(1+\sup _{\sigma \in \mathbb{R}}\|u(\sigma)\|_{X^{1+\epsilon}}^{\rho}\right) \frac{1}{a^{\gamma(\epsilon)-\theta}} .
$$


Step 3. Let

$$
B(r):=\left\{w \in P A A\left(X^{1+\epsilon}, \mu\right): \sup _{t \in \mathbb{R}}\|w(t)\|_{X^{1+\epsilon}} \leq r\right\} .
$$

We claim that $T(B(r)) \subset B(r)$. Indeed, for $u \in B(r)$ we have

$$
\begin{aligned}
\|(T u)(t)\|_{X^{1+\epsilon}} & \leq M \int_{-\infty}^{t} e_{\alpha, \alpha(\gamma(\epsilon)-\epsilon)}^{\gamma(\epsilon)-\epsilon}(t-s, a)\|f(s, u(s))\|_{X^{\gamma(\epsilon)}} d s \\
& \leq \frac{M c\left(1+r^{\rho}\right)}{a^{\gamma(\epsilon)-\epsilon}}<r .
\end{aligned}
$$

Next, we prove that $T$ is a contraction. Let $u, v \in B(r)$. Then

$$
\begin{aligned}
& \|(T u)(t)-(T v)(t)\|_{X^{1+\epsilon}} \leq M \int_{-\infty}^{t} e_{\alpha, \alpha(\gamma(\epsilon)-\epsilon)}^{\gamma(\epsilon)-\epsilon}(t-s, a)\|f(s, u(s))-f(s, v(s))\|_{X^{\gamma(\epsilon)}} d s \\
& \leq M c \int_{-\infty}^{t} e_{\alpha, \alpha(\gamma(\epsilon)-\epsilon)}^{\gamma(\epsilon)-\epsilon}(t-s, a)\left(1+\|u(s)\|_{X^{1+\epsilon}}^{\rho-1}+\|v(s)\|_{X^{1+\epsilon}}^{\rho-1}\right)\|u(s)-v(s)\|_{X^{1+\epsilon}} d s \\
& \leq \frac{M c\left(1+2 r^{\rho-1}\right)}{a^{\gamma(\epsilon)-\epsilon}} \sup _{t \in \mathbb{R}}\|u(t)-v(t)\|_{X^{1+\epsilon}} .
\end{aligned}
$$

Therefore $T$ is a contraction. The conclusion follows from Fixed Point Theorem.

Example 4.6. Let $\Omega \subset \mathbb{R}^{N}$ be a bounded domain with smooth boundary. Let $0<\alpha<1, \rho>1$. We consider the following heat equation with Dirichlet boundary conditions

$$
\begin{cases}D_{t}^{\alpha} u=A u+g h(u), & \text { in } \mathbb{R} \times \Omega, \\ u=0 & \text { on } \partial \Omega\end{cases}
$$

where $g \in P A A(\mathbb{R}, \mu), h(u)=u|u|^{\rho-1}$ and $A=\Delta-a$, with $a>0$, on $E_{q}^{0}:=L^{q}(\Omega)$, where $q=\frac{N(\rho-1)}{2}$, defined on

$$
D(A)=W^{2, q}(\Omega) \cap W_{0}^{1, q}(\Omega) .
$$

We denote the fractional power spaces associated to $A$ by $\left\{E_{q}^{\beta}\right\}_{\beta \in \mathbb{R}}$. Let $A_{\beta}$ be the realization of $A$ in $E_{q}^{\beta}$. Then it is well known that

$$
-A_{\beta}: D\left(A_{\beta}\right)=E_{q}^{\beta+1} \subset E_{q}^{\beta} \rightarrow E_{q}^{\beta}
$$

is a-a-sectorial operator. Define

$$
X_{q}^{\beta}:=E_{q}^{\beta-1}, \quad \beta \in \mathbb{R} .
$$

Let $f: \mathbb{R} \times X_{q}^{\gamma(\epsilon)} \rightarrow X_{q}^{1+\epsilon}$ given by $f(t, u)=g(t) u|u|^{\rho-1}$. If $1<q<\frac{N}{N-2}$, then by $[5$, Lemma 8] we have that $f$ is $\epsilon$-regular map relative to $\left(X_{q}^{1}, X_{q}^{0}\right)$ for $0<\epsilon_{0}(q)<\epsilon<\frac{N}{N+2 q}$, with $\epsilon_{0}(q)=$ $\frac{N}{N+2 q}\left(1-\frac{N}{2}\left(1-\frac{1}{q}\right)\right)$ and $\gamma(\epsilon)=\rho \epsilon$. It can be easily be checked that

$$
f \in \mathcal{F}(\epsilon, \gamma(\varepsilon), \rho, c) \cap P A A_{U}\left(X_{q}^{1+\epsilon}, X_{q}^{\gamma(\epsilon)}, \mu\right) .
$$

If the constants $M$ and $c$ are as in Theorem 4.5 then there exists a unique $\epsilon$-mild regular solution $u \in P A A\left(X_{q}^{1+\theta}, \mu\right)$ of (4.5) for every $0 \leq \theta<\gamma(\epsilon)$. 


\section{APPENDIX}

In this appendix we provide some definitions and results about the theory of $\mu$-pseudo almost automorphic functions which are used along the paper, in order to simplify the reading.

Let $X, Y$ be Banach spaces with norms $\|\cdot\|$ and $\|\cdot\|_{Y}$, respectively. We denote by $B C(X)$ the Banach space of bounded continuous functions from $\mathbb{R}$ to $X$, equipped with the supremum norm $\|f\|_{\infty}=\sup _{t \in \mathbb{R}}\|f(t)\|$.

Definition 5.1. ([20]) A continuous function $f: \mathbb{R} \rightarrow X$ is said almost automorphic if for every sequence of real numbers $\left\{s_{n}^{\prime}\right\}_{n \in \mathbb{N}}$ there exists a subsequence $\left\{s_{n}\right\}_{n \in \mathbb{N}}$ such that

$$
g(t):=\lim _{n \rightarrow \infty} f\left(t+s_{n}\right)
$$

is well defined for each $t \in \mathbb{R}$, and

$$
\lim _{n \rightarrow \infty} g\left(t-s_{n}\right)=f(t)
$$

for each $t \in \mathbb{R}$. The collection of all such functions will be denoted by $A A(X)$.

Example 5.2. ([21, Example 2.1]) Let $\kappa: \mathbb{R} \rightarrow \mathbb{R}$ such that

$$
\kappa(t)=\cos \left(\frac{1}{2-\sin \pi t-\sin t}\right), \text { for } t \in \mathbb{R} .
$$

Then $\kappa$ is an almost automorphic function, but it is not uniformly continuous on $\mathbb{R}$.

We recall the notion of $\mu$-pseudo almost automorphic functions under the light of measure theory given in [10], which is a generalization of the pseudo almost automorphy.

Let $\mathcal{B}$ be the Lebesgue $\sigma$-field of $\mathbb{R}, \mathcal{M}$ the set of all positive measure $\mu$ on $\mathcal{B}$ satisfying $\mu(\mathbb{R})=\infty$ and $\mu([a, b])<\infty$ for all $a, b \in \mathbb{R}$ with $a \leq b$.

Definition 5.3. ([10, Def. 2.5]) Let $\mu \in \mathcal{M}$. A function $f \in B C(X)$ is called $\mu$-ergodic if

$$
\lim _{T \rightarrow+\infty} \frac{1}{\mu([-T, T])} \int_{[-T, T]}\|f(t)\| d \mu(t)=0 .
$$

We denote by $\mathcal{E}(X, \mu)$ the set of such functions.

Proposition 5.4. ([10, Prop. 2.13]) Let $\mu \in \mathcal{M}$. Then $\mathcal{E}(X, \mu)$ is a Banach space with respect to the supremum norm.

Definition 5.5. ([10, Def. 2.6]) Let $\mu \in \mathcal{M}$. A continuous function $f: \mathbb{R} \rightarrow X$ is called $\mu$-pseudo almost automorphic if it can be decomposed as $f=g+\varphi$, where $g \in A A(X)$ and $\varphi \in \mathcal{E}(X, \mu)$. We denote by $P A A(X, \mu)$ the collection of such functions.

Theorem 5.6. ([10, Th. 3.5]) Let $\mu \in \mathcal{M}$ satisfying the hypothesis

(H) For all $\tau \in \mathbb{R}$, there exist $\beta>0$ and a bounded interval $I$ such that $\mu(\{a+\tau, a \in A\}) \leq$ $\beta \mu(A)$ if $A \in \mathcal{B}$ satisfies $A \cap I=\emptyset$.

Then $\mathcal{E}(X, \mu)$ is translation invariant, and therefore $P A A(X, \mu)$ is also translation invariant.

Remark 5.7. (a) If the measure $\mu$ is the Lebesgue measure, then the space $P A A(X, \mu)$ coincides with the space of the pseudo almost automorphic functions $P A A(X)$ ([46]). 
(b) Let $\rho(t)>0$ a.e on $\mathbb{R}$ for the Lebesgue measure, locally-Lebesgue integrable on $\mathbb{R}$ and $\int_{\mathbb{R}} \rho(t) d t=\infty$. Let $\mu$ be the positive measure defined by

$$
\mu(A)=\int_{A} \rho(t) d t, \quad \text { for } A \in \mathcal{B},
$$

where $d t$ denotes the Lebesgue measure on $\mathbb{R}$. Then $P A A(X, \mu)$ coincides with the space of weighted pseudo almost periodic functions $W P A A(X)$ ([9]).

Theorem 5.8. ([10, Theorem 4.9]) Let $\mu \in \mathcal{M}$. Assume that $P A A(X, \mu)$ is translation invariant. Then $P A A(X, \mu)$ is a Banach space with the supremum norm.

Definition 5.9. ([8]) A continuous function $f: \mathbb{R} \times Y \rightarrow X$ is said almost automorphic in $t$ uniformly with respect to $y$ in $Y$ if the two following conditions hold:

(a) $f(\cdot, y) \in A A(X)$ for all $y \in Y$.

(b) $f$ is uniformly continuous on each compact $K \subset Y$ with respect to the second variable $y$.

We denote by $A A_{U}(Y, X)$ the set of such functions.

Theorem 5.10. ([14, Theorem 3.5]) Let $f \in A A_{U}(Y, X)$. If $u \in A A(Y)$, then $f(\cdot, u(\cdot)) \in$ $A A(X)$.

Definition 5.11. ([10, Def. 5.3]) Let $\mu \in \mathcal{M}$. A continuous function $f: \mathbb{R} \times Y \rightarrow X$ is called $\mu$-ergodic in $t$ uniformly with respect to $y \in Y$ if the following two conditions hold:

(a) $f(\cdot, y) \in \mathcal{E}(X, \mu)$ for all $y \in Y$.

(b) $f$ is uniformly continuous on each compact set $K \subset Y$ with respect to the second variable $y$.

Denote by $\mathcal{E}_{U}(Y, X, \mu)$ the set of such functions.

Definition 5.12. ([10, Def. 5.4]) Let $\mu \in \mathcal{M}$. A continuous function $f: \mathbb{R} \times Y \rightarrow X$ is called $\mu$-pseudo almost automorphic in $t$ uniformly with respect to $y \in Y$ if $f=g+\phi$ where $g \in A A_{U}(Y, X)$ and $\phi \in \mathcal{E}_{U}(Y, X, \mu)$. Denote by $P A A_{U}(Y, X, \mu)$ the set of such functions.

Theorem 5.13. ([10, Th. 5.7]) Let $\mu \in \mathcal{M}, f \in P A A_{U}(Y, X, \mu)$ and $u \in P A A(Y, \mu)$. Assume that the following hypothesis holds

(C) For all bounded subset $B$ of $Y, f$ is bounded on $\mathbb{R} \times B$.

Then $t \mapsto f(t, u(t)) \in P A A(X, \mu)$.

\section{ACKNOWLEDGMENTS}

This paper was written while the second named author was visiting the Departments of Mathematics of the University of Zaragoza and the CUD (Spain). He wishes to thank the departments for the generous hospitality, in particular to Professor L. Abadias.

\section{REFERENCES}

[1] L. Abadias, E. Alvarez and C. Lizama, Regularity properties of mild solutions for a class of volterra equation with critical nonlinearities, J. Integral Equations Appl. (to appear).

[2] L. Abadias, C. Lizama and P. J. Miana, Sharp extensions and algebraic properties for solutions families of vector-valued differential equations, Banach J. Math. Anal. 10 (2016), 169-208. 
[3] L. Abadias and P. J. Miana, A subordination principle on Wright functions and regularized resolvent families, J. Funct. Spaces. Art. ID 158145, 9 pages, (2015).

[4] W. Arendt, C. J. K. Batty, M. Hieber and F. Neubrander, Vector-valued Laplace transforms and Cauchy problems, Second edition, Monographs in Mathematics, vol 96, Birkhäuser, 2011.

[5] J. M. Arrieta and A. N. Carvalho, Abstract parabolic problems with critical nonlinearities and applications to Navier-Stokes and heat equations, Trans. Amer. Math. Soc. 352 (1) (1999), 285-310.

[6] E. Bajlekova, Subordination principle for fractional evolution equations, Fract. Calc. Appl. Anal. 3 (3) (2000), 213-230.

[7] E. Bajlekova, Fractional Evolution Equations in Banach Spaces, Ph.D. Thesis, University Press Facilities, Eindhoven University of Technology 2001.

[8] J. Blot, P. Cieutat, G. M. N'Guérékata and D. Pennequin, Superposition operators between various almost periodic function spaces and applications, Commu. in Math. Anal. 6 (1) (2009), 42-70.

[9] J. Blot, G. M. Mophou, G. M. N'Guérékata and D. Pennequin, Weighted pseudo almost automorphic functions and applications to abstract differential equations, Nonlinear Anal. 71 (2009), 903-909.

[10] J. Blot, P. Cieutat, and K. Ezzinbi, Measure theory and pseudo almost automorphic functions: New developments and applications, Nonlinear Anal. 75 (4) (2012), 2426-2447.

[11] S. Bochner, Diffusion equation and stochastic processes, Proc. Nat. Acad. Sci. USA 35 (1949), 368-370.

[12] E. Capelas de Oliveira, F. Mainardi, and J. Vaz Jr, Models based on Mittag-Leffler functions for anomalous relaxation in dielectrics, Eur. Phys. J. Special Topics, 193:161-171, 2011. Revised version in http://arxiv.org/abs/1106.1761.

[13] C. Chen and M. Li, On fractional resolvent operator functions, Semigroup Forum. 80 (2010), 121-142.

[14] P. Cieutat, S. Fatajou and G. M. N'Guérékata, Composition of pseudo almost periodic and pseudo almost automorphic functions and applications to evolution equations, Appl. Anal. 89 (1) (2010), 11-27.

[15] B. De Andrade, A. N. Carvalho, P. M. Carvalho-Neto and P. Marín-Rubio, Semilinear fractional differential equations: Global solutions, critical nonlinearities and comparison results, Topol. Methods Nonlinear Anal. 45 (2) (2015), 439-467.

[16] B. De Andrade, C. Cuevas, J. Liang and H. Soto, Periodicity and ergodicity for abstract evolution equations with critical nonlinearities, Adv. in. Difference Eq. 2015 (2015), 1-20.

[17] K. J. Engel and R. Nagel, One-Parameter semigroups for linear evolution equations, Graduate texts in Mathematics, vol 194, Springer-Verlag, New York, 2000.

[18] R. Figueiredo Camargo, A. O. Chiacchio, R. Charnet and E. Capelas de Oliveira, Solution of the fractional Langevin equation and the Mittag-Leffler functions, Journal of Mathematical Physics. 50 (2009), 1-8.

[19] R. Gorenflo, A. A. Kilbas, F. Mainardi and S. V. Rogosin, Mittag-Leffler functions, related topics and applications, Springer Monographs in Mathematics, Springer, Heidelberg, 2014.

[20] G. M. N'Guérékata, Almost automorphic and almost periodic functions in abstract spaces, Kluwer, New York, 2001.

[21] G. M. N'Guérékata and A. Pankov, Stepanov-like almost automorphic functions and monotone evolution equations, Nonlinear Anal. 68 (2008), 2658-2667.

[22] B. H. Guswanto and T. Suzuki, Existence and uniqueness of mild solutions for fractional semilinear differential equations, Electron. J. Diff. Equ. 168 (2015), 1-16.

[23] M. Haase, The functional calculus for sectorial operators, Operator Theory: Advances and Applications, vol 169, Birkhäuser, Basel, 2006.

[24] A. Hanyga and M. Seredyńska, On a mathematical framework for the constitutive equations of anisotropic dielectric relaxation, J. Stat. Phys. 131 (2008), 269-303.

[25] D. Henry, Geometric theory of semilinear parabolic equations, Lecture Notes in Mathematics, vol 840, Springer-Verlag, Berlin, 1981.

[26] B. Kaltenbacher and I. Lasiecka, Wellposedness and exponential decay rates for the Moore-Gibson-Thompson equation arising in high intensity ultrasound, Control Cybernet. 40 (4) (2011), 971-988.

[27] A. A. Kilbas, H. M. Srivastava and J. J. Trujillo, Theory and applications of fractional differential equations, North-Holland Mathematics Studies, vol 204, Elsevier Science B.V., Amsterdam, 2006.

[28] M. Kostić, $(a, k)$-regularized C-resolvent families: regularity and local properties, Abstr. Appl. Anal. (2009), Article ID 858242, 27 pages.

[29] Y. N. Li and H. R. Sun, Integrated fractional resolvent operator function and fractional abstract Cauchy problem, Abstr. Appl. Anal. (2014), Article ID 430418, 9 pages.

[30] K. Li and J. Peng, Fractional resolvents and fractional evolution equations, Appl. Math. Lett. 25 (2012) 808-812. 
[31] C. Lizama, Regularized solutions for abstract Volterra equations, J. Math. Anal. Appl. 243 (2000), 278-292.

[32] C. Lizama and E. Vergara, Uniform stability of resolvent families, Proc. Amer. Math. Soc. 132 (1) (2004), 175-181.

[33] F. Mainardi, Fractional calculus and waves in linear viscoelasticity, an introduction to mathematical models, Imperial College Press, 2010.

[34] F. Mainardi, G. Pagnini and R. Gorenflo, Mellin transform and subodination laws in fractional diffusion processes, Fract. Calc. Appl. Anal. 6 (4) (2003) 441-459.

[35] Z-D. Mei, J-G. Peng and Y. Zhang, A characteristic of fractional resolvents, Fract. Calc. Appl. Anal. 16 (4) (2013), 777-790.

[36] K. S. Miller and B. Ross, An introduction to the fractional calculus and fractional differential equations, Wiley, New York, 1993.

[37] J. Mu, Y. Zhou and L. Peng, Periodic Solutions and S-Asymptotically Periodic Solutions to Fractional Evolution Equations, Discrete Dyn. Nat. Soc. (2017), Article ID 1364532, 12 pages.

[38] D. Mugnolo, Asymptotic of semigroups generated by operator matrices, Arab. J. Math. 3 (2014), 419-435.

[39] R.R. Nigmatullin, The realization of the generalized transfer equation in a medium with fractal geometry, Phys. Status Sol. (B). 33 (1986), 425-430.

[40] A. Pazy, Semigroups of linear operators and applications to partial differential equations, Springer-Verlag, New York, 1983.

[41] T. R. Prabhakar, A singular integral equation with a generalized Mittag-Leffler function in the kernel, Yokohama Math. J. 19 (1971), 7-15.

[42] I. Podlubny, Fractional differential equations, Vol. 98 of Mathematics in Science and Engineering, Acamedic Press, San Diego, Calif., USA, 1999.

[43] J. Prüss, Evolutionary integral equations and applications, Modern Birkhäuser Classics, Birkhäuser/Springer, Basel, 2012.

[44] J. V. Neerven, The asymptotic behaviour of semigroups of linear operators, Vol. 88, Operator Theory Advances and Applications, Birkhäuser, 1996.

[45] E. M. Wright, On the coefficients of power series having exponential singularities, J. London Math. Soc. 8 (1933), 71-79

[46] T-J. Xiao, J. Liang and J. Zhang, Pseudo almost automorphic solutions to semilinear differential equations in Banach spaces, Semigroup Forum. 76 (2008), 518-524.

[47] K. Yosida, Functional Analysis, Fifth edition, A Series of Comprehensive Studies in Mathematics, vol 123, Springer, 1978.

(L. Abadias) Departamento de Matemáticas, Instituto Universitario de Matemáticas y Aplicaciones, Universidad DE ZaragoZA, 50009 ZaragozA, Spain.

E-mail address: labadias@unizar.es

(E. Álvarez) Departamento de Matemáticas y Estadística, Universidad del Norte, Barranquilla, Colombia

E-mail address: ealvareze@uninorte.edu.co 